\title{
Prevalence and Risk Factors of Diabetes Mellitus in Patients with Autoimmune Hepatitis
}

\author{
Naoki Matsumoto, Masahiro Ogawa, Shunichi Matsuoka and Mitsuhiko Moriyama
}

\begin{abstract}
Objective The administration of corticosteroids is a standard treatment for autoimmune hepatitis (AIH), but it can occasionally induce various adverse effects. Diabetes mellitus (DM) is a major complication of chronic liver diseases. We investigated the prevalence and risk factors of DM in patients with AIH.

Methods We retrospectively analyzed 118 Japanese patients diagnosed with AIH from 1990 to 2014 at our institution. The prognosis of patients with and without DM was also compared.

Results Twenty-nine (24.5\%) patients had DM and 21 (72.4\%) received corticosteroids. The annual cumulative incidence rate of newly diagnosed DM was $1.2 \%$. Multivariate analysis showed that DM occurred in older patients $[\mathrm{OR}=6.290 ; 95 \%$ confidence interval $(\mathrm{CI})=1.230-32.100 ; \mathrm{p}=0.018]$ with higher serum immunoglobulin $\mathrm{G}$ levels $(\mathrm{OR}=12.400 ; 95 \% \mathrm{CI}=2.560-60.400 ; \mathrm{p}=0.002)$. A Cox hazard regression analysis revealed that predictive factors for $\mathrm{DM}$ were absence of other autoimmune diseases $(\mathrm{OR}=0.171 ; 95 \% \mathrm{CI}=0.036-0.805$; $\mathrm{p}=0.025)$, use of corticosteroids $(\mathrm{OR}=6.693$; 95\% $\mathrm{CI}=1.391-32.210 ; \mathrm{p}=0.049)$ and lower platelet counts $(\mathrm{OR}=$ $3.873 ; 95 \% \mathrm{CI}=1.021-14.690 ; \mathrm{p}=0.046)$. The 10 year survival rates of the DM and non-DM groups were $94.1 \%$ and $94.6 \%$, respectively. There was no significant difference between these groups ( $\mathrm{p}=0.293$ ).

Conclusion DM occurred in $24.5 \%$ of patients with $\mathrm{AIH}$; older age, absence of other autoimmune diseases and higher serum immunoglobulin $\mathrm{G}$ levels are risk factors. Taking corticosteroids and a lower platelet count are risk factors for a new onset of DM. DM did not influence the prognosis of AIH patients.
\end{abstract}

Key words: autoimmune hepatitis, diabetes mellitus, corticosteroid

(Intern Med 55: 879-885, 2016)

(DOI: 10.2169/internalmedicine.55.4957)

\section{Introduction}

Autoimmune hepatitis (AIH) is a chronic liver disease that was first reported by Waldenström in 1950 (1). If left untreated, AIH progresses to liver cirrhosis and liver failure, whereas it has a relatively good prognosis if the patient is treated appropriately $(2,3)$. The standard treatment for AIH is immunosuppressive therapy including corticosteroids and azathioprine (2, 4-6). However, corticosteroids can cause adverse effects including diabetes mellitus (DM), immunosuppression, osteoporosis, hypertension, cataracts and glaucoma (7).

Diabetes mellitus is a major complication of chronic liver diseases $(8,9)$ and should be considered because it is a risk factor for mortality in patients with chronic liver disease as- sociated with cardiovascular disease, malignancy and liverrelated death (10-12). A population-based study indicates that DM increases the overall mortality rate 2.3-30.8-fold in patients with chronic hepatitis $\mathrm{B}$, alcoholic liver disease and non-alcoholic fatty liver disease (NAFLD) (11). Although there are many reports regarding the prevalence of DM in patients with hepatitis C virus (HCV) infection (13-17), hepatitis B virus (HBV) infection (18), alcoholic liver disease $(11,19)$ and $\operatorname{NAFLD}(11,20)$, the incidence rate of DM in AIH has not been reported. The involvement of DM in HCV-infected patients is correlated with hepatic functional reserve $(21,22)$. Moreover, the risk factors of DM in patients with AIH are also unknown.

This retrospective study aimed to clarify the prevalence and risk factors of DM in patients with AIH. 
Table 1. Patients Characteristics of DM Group and Non-DM Group.

\begin{tabular}{lccc}
\hline & AIH with DM & AIH without DM & p value \\
\hline $\mathrm{n}$ & 29 & 89 & \\
Gender (Male/Female) & $6 / 23$ & $5 / 84$ & 0.025 \\
Age (years) & $60(43 \sim 80)$ & $54(11 \sim 75)$ & 0.004 \\
Cirrhosis at diagnosis & $5(17.2 \%)$ & $7(7.9 \%)$ & 0.165 \\
Develop cirrhosis & $5(20.0 \%)$ & $7(8.6 \%)$ & 0.149 \\
Relapse & $8(29.6 \%)$ & $26(30.6 \%)$ & 1 \\
Fibrosis stage (F3-4/F0-2) & $14 / 9$ & $51 / 9$ & 0.034 \\
Other autoimmune disease & $5(17.2 \%)$ & $39(43.8 \%)$ & 0.014 \\
Malignant tumor & $3(10.3 \%)$ & $7(7.8 \%)$ & 0.706 \\
Use of Corticosteroids & $21(72.4 \%)$ & $48(53.9 \%) *$ & 0.127 \\
Body Mass Index & $22.8(18.4 \sim 32.9)$ & $23.4(15.9 \sim 30.5)$ & 0.952 \\
Baseline laboratory values & & & \\
AST $(<38$ IU/L) & $178(21 \sim 1,369)$ & $103(14 \sim 19,350)$ & 0.734 \\
ALT $(<45$ IU/L) & $148(14 \sim 1,951)$ & $114(8 \sim 10,680)$ & 0.868 \\
ALP $(<335$ IU/L) & $379(138 \sim 1,329)$ & $434(130 \sim 14,016)$ & 0.953 \\
Bilirubin $(<1.1$ mg/mL) & $1.0(0.4 \sim 18.2)$ & $0.8(0.2 \sim 26.9)$ & 0.128 \\
Albumin $(3.5-5.0$ g/L) & $3.9(2.3 \sim 5.2)$ & $4.1(2.9 \sim 5.1)$ & 0.306 \\
IgG $(500-1,300$ mg/dL) & $2,707(1,481 \sim 4,820)$ & $1,840(615 \sim 4,503)$ & $<0.001$ \\
Platelets $(15-40 \times 10 / \mu L)$ & $14.9(2.3 \sim 29.8)$ & $19.7(6.7 \sim 39.4)$ & 0.021 \\
ANA+( $\geq 1: 40)$ & $20(71.4 \%)$ & $50(64.1 \%)$ & 0.642 \\
\hline DM: diabetes mellitus, AST: aspartate amino transferase, ALT: alanine minotransferase, \\
ALP: alkaline phosphatase, IgG: immunoglobulin G, ANA: Anti-nuclear antibodies \\
Data of relapse was not availablein six cases. & &
\end{tabular}

\section{Materials and Methods}

\section{Study population}

A total of 118 consecutive patients diagnosed with AIH from 1990 to 2014 at our institution were enrolled in this retrospective study. All patients met the 1999 revised criteria of the International Autoimmune Hepatitis Group for a diagnosis of definite AIH (45 cases) or probable AIH diagnosed with a biopsy (73 cases) (23). All patients underwent abdominal ultrasonography, and only one case was diagnosed as fatty liver disease without the use of a liver biopsy. Patients positive for hepatitis B surface antigen or HCV antibody and those that consumed too much alcohol were excluded. The patients were compared with those in a control group. The characteristics of the patients meeting the inclusion criteria are shown in Table 1. The follow-up period ranged from 0 to 28 years (median, 8.8 years). Autoimmune disease-related complications included chronic thyroiditis in 14 cases, rheumatoid arthritis in eight cases, Sjögren's syndrome in eight cases and systemic lupus erythematosus in seven cases. There were only two cases of AIH-primary biliary cirrhosis overlap syndrome.

This study was performed in accordance with the principles of the Declaration of Helsinki. Written informed consent regarding the use of the data was obtained from each subject. Clinical data including serological and follow-up data were obtained from the hospital records for all cases. This study was approved by the Institutional Review Board of our hospital (approval number: RK-150414-2).

\section{Clinical and histological assessments}

The following factors were examined: gender, age, AIH score, aspartate amino transferase (AST), alanine aminotransferase (ALT), alkaline phosphatase (ALP), bilirubin, albumin, platelet count and immunoglobulin $G$ at the time of AIH diagnosis. Plasma glucose and hemoglobin A1c (HbA1c) were examined during the diagnosis of $\mathrm{AIH}$ and every three years thereafter. Anti-nuclear antibodies and smooth muscle antibodies were measured by indirect immunofluorescence, and cutoff titers were 1:40. AIH-primary biliary cirrhosis overlap syndrome was diagnosed based on previously reported criteria (24). A diagnosis of liver cirrhosis was made based on a needle biopsy or imaging findings, combined with the use of serum markers (25).

Liver tissue specimens were obtained by a needle biopsy in 83 of the 118 patients $(70.3 \%)$. Histological assessments were performed by two pathologists. Liver fibrosis was evaluated using the METAVIR scoring system (26). Fibrosis was rated on a $0-4$ scale as follows: $\mathrm{F} 0=$ no fibrosis; $\mathrm{F} 1=$ portal fibrosis without septa; F2= portal fibrosis and a few septa; F3= numerous septa without cirrhosis; F4= cirrhosis.

\section{Diabetes mellitus diagnosis}

DM was diagnosed when a patient had a fasting plasma glucose level of $126 \mathrm{mg} / \mathrm{dL}$ or greater, had a non-fasting plasma glucose level of $200 \mathrm{mg} / \mathrm{dL}$ or greater or had an HbA1c level of $6.5 \%$ or greater. The patients were also considered to have diabetes if they used insulin or hypoglycemic drugs at the time of the survey. The values for HbAlc were unified with the National Glycohemoglobin Standardization Program (NGSP) values. The Japanese Diabetes Soci- 
Table 2. Logistic Regression Analysis of the Predictive Factors for DM in AIH.

\begin{tabular}{lcccc}
\hline & \multicolumn{2}{c}{ Univariate analysis } & Multivariate analysis \\
& OR $(95 \% \mathrm{CI})$ & $\mathrm{p}$ & OR $(95 \% \mathrm{CI})$ & $\mathrm{p}$ \\
\hline Gender (Male/Female) & $2.220(0.435-11.400)$ & 0.337 & & \\
Age (years, $\geq 50 /<50)$ & $4.420(0.751-26.000)$ & 0.100 & $6.290(1.230-32.100)$ & 0.018 \\
Fibrosis stage $(\mathrm{F} 3-4 / \mathrm{F} 0-2)$ & $2.510(0.592-10.700)$ & 0.212 & & \\
Other autoimmune disease & $0.490(0.123-1.950)$ & 0.311 & & \\
Use of Corticosteroids & $0.868(0.215-3.510)$ & 0.843 & & \\
Baseline laboratory values & & & & \\
IgG (mg/dL, $\geq 1,860 /<1,860)$ & $7.550(1.880-30.200)$ & 0.004 & $12.400(2.560-60.400)$ & 0.002 \\
Platelets $\left(10^{4} / \mu \mathrm{L}, \geq 18 /<18\right)$ & $0.964(0.240-3.870)$ & 0.959 & & \\
\hline DM: diabetes mellitus, IgG: immunoglobulin $\mathrm{G}$ & & &
\end{tabular}

DM: diabetes mellitus, IgG: immunoglobulin G

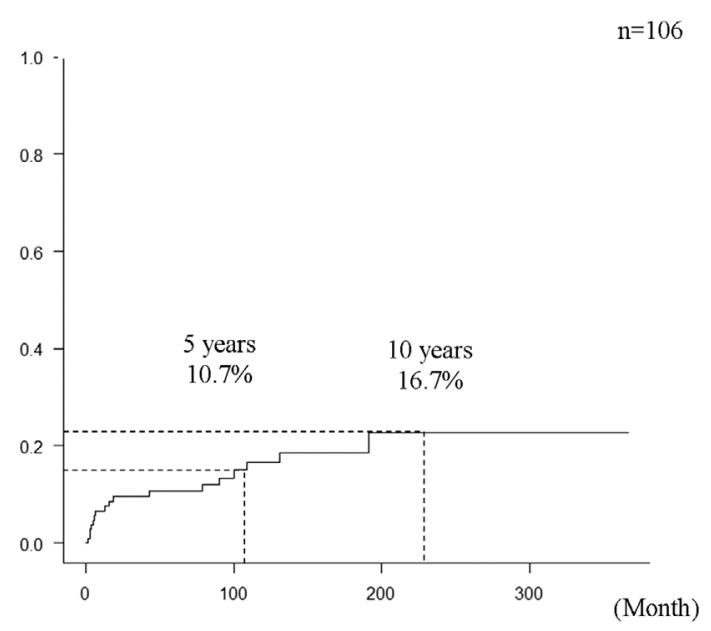

Figure 1. Cumulative rate of newly diagnosed DM patients with AIH. The incidence rate was $10.7 \%$ at five years, $16.7 \%$ at 10 years and $1.2 \%$ per year.

ety (JDS) values were converted to NGSP values before 2012. Type 1 DM was diagnosed according to the American Diabetes Association criteria or the presence of insulin dependence, weight loss and polyuria.

\section{Statistical analysis}

The results are presented as the means \pm standard deviation or as numbers. The significance of the differences in quantitative data was determined using the Mann-Whitney $U$-test, Fisher's exact probability test or multivariate logistic regression analysis. A multivariate analysis for diabetes was carried out by logistic regression. The cumulative development rates of malignancies were calculated using the Kaplan-Meier technique, and differences in the curves were tested using the log-rank test. The Statistical Program for Social Sciences software package (SPSS 11.5 for Windows, SPSS, Chicago, USA) was used to perform all statistical analyses. The level of significance was set at $\mathrm{p}<0.05$.

\section{Results}

\section{Prevalence and risk factors of diabetes mellitus}

The total number of patients with DM in this study was
$29(24.5 \%)$, and $21(72.4 \%)$ of them received corticosteroids. There was only one patient with type $1 \mathrm{DM}$. The patient was a 62-year-old man who tested negative for the presence of an anti-nuclear antibody. His anti-M2 of the anti-mitochondrial antibody (index) was 55.4. During the diagnosis of type $1 \mathrm{DM}$, the patient's anti-glutamic acid decarboxylase antibody level was $17.5 \mathrm{U} / \mathrm{mL}$.

A comparison between patients with and without DM revealed that the DM group was older $(p=0.004)$ and consisted of more men $(\mathrm{p}=0.025)$ than the non-DM group. Additionally, the absence of other autoimmune diseases $(\mathrm{p}=$ $0.014)$ and F3-4 (p=0.034) were more common in the DM group. Moreover, the DM group had a significantly higher serum immunoglobulin $\mathrm{G}$ level $(\mathrm{p}<0.001)$, and a lower platelet count $(\mathrm{p}=0.021)$ than did the non-DM group (Table 1).

Multivariate analysis showed that DM occurred in older patients $[\mathrm{OR}=6.290 ; 95 \%$ confidence interval $(\mathrm{CI})=1.230$ $32.100 ; \mathrm{p}=0.018$ ] with higher serum immunoglobulin $\mathrm{G}$ levels $(\mathrm{OR}=12.400 ; 95 \% \mathrm{CI}=2.560-60.400 ; \mathrm{p}=0.002)$ (Table 2).

\section{Development of DM during the observation period}

During the observation period, DM developed in 17 of 106 patients $(16.0 \%)$. The cumulative incidence rate of newly diagnosed DM was $1.2 \%$ per year $(10.7 \%$ and $16.7 \%$ at 5 and 10 years, respectively) (Fig. 1). Of these patients, $76.5 \%$ started taking corticosteroids. The mean fasting plasma glucose level during the diagnosis of AIH and 3, 6, 9,12 , and 15 years later was $108.0 \pm 44.4,119.0 \pm 34.2$, $128.0 \pm 25.4,117.0 \pm 25.4,148.0 \pm 37.7$ and $135.0 \pm 17.4 \mathrm{mg} / \mathrm{dL}$, respectively. The mean HbA1c level ( 5 LC cases were excluded) was $6.0 \pm 1.0,6.1 \pm 0.8,6.1 \pm 0.6,6.3 \pm 0.3,6.3 \pm 0.7$ and $6.3 \pm 0.6 \%$, respectively (Fig. 2, 3). An analysis of fasting plasma glucose and HbA1c levels revealed no difference from the time of diagnosis to 15 years later.

A Cox hazard regression analysis revealed that predictive factors for DM were absence of other autoimmune diseases $(\mathrm{OR}=0.171 ; 95 \% \mathrm{CI}=0.036-0.805 ; \mathrm{p}=0.025)$, use of corticosteroids $(\mathrm{OR}=6.693 ; 95 \% \mathrm{CI}=1.391-32.210 ; \mathrm{p}=0.049)$ and a lower platelet count $(\mathrm{OR}=3.873 ; 95 \% \mathrm{CI}=1.021-14.690$; $\mathrm{p}=0.046$ ) (Table 3). 


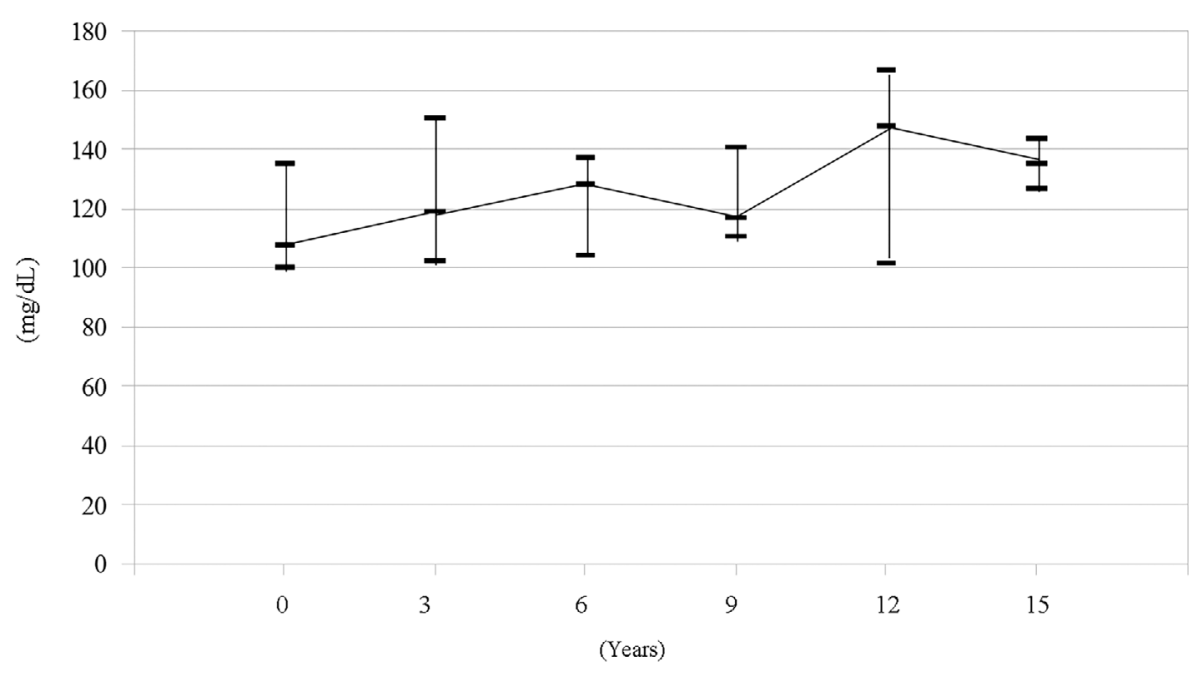

Figure 2. Mean fasting plasma glucose during diagnosis of AIH and 3, 6, 9, 12, and 15 years later. The results revealed no significant difference during the 15 years following diagnosis.

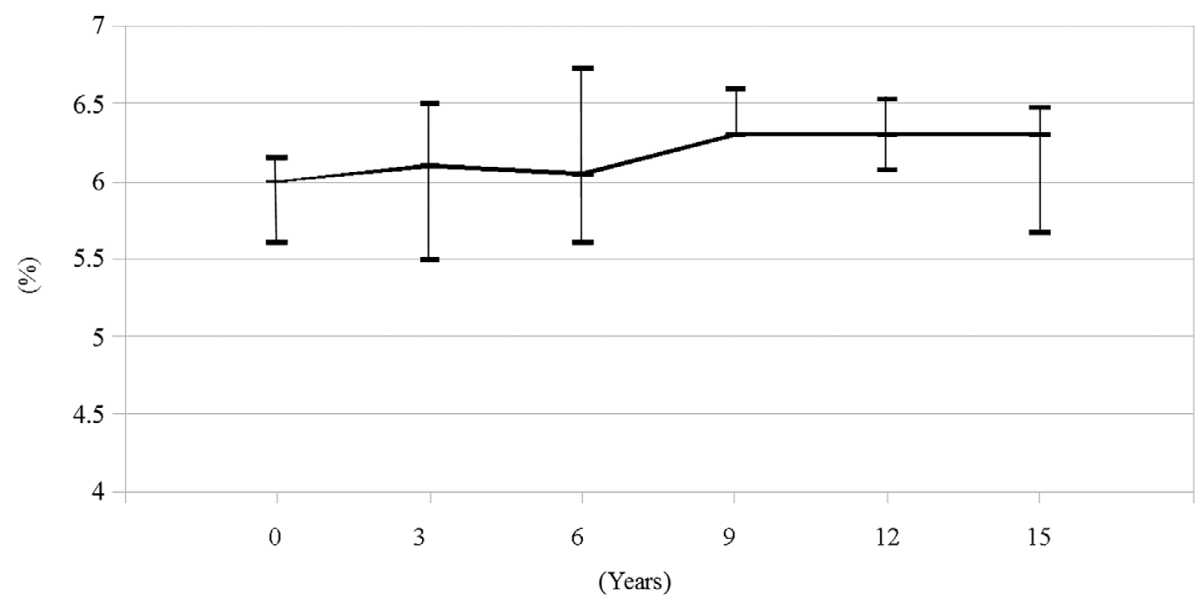

Figure 3. Mean HbA1c during diagnosis of AIH and 3, 6, 9, 12 and 15 years later. The results revealed no significant difference during the 15 years following diagnosis.

Table 3. Cox Hazard Regression Analysis of the Predictive Factors for DM Onset in AIH.

\begin{tabular}{lcccc}
\hline & Univariate analysis & \multicolumn{2}{c}{ Multivariate analysis } \\
& OR $(95 \% \mathrm{CI})$ & $\mathrm{p}$ & OR $(95 \% \mathrm{CI})$ & $\mathrm{p}$ \\
\hline Gender (Male/Female) & $1.764(0.323-9.646)$ & 0.513 & & \\
Age (years, $\geq 50 /<50)$ & $2.417(0.367-15.910)$ & 0.359 & & \\
Fibrosis stage (F3-4/F0-2) & $1.911(0.507-7.198)$ & 0.339 & & \\
Other autoimmune disease & $0.367(0.071-1.882)$ & 0.229 & $0.171(0.036-0.805)$ & 0.025 \\
Use of Corticosteroids & $1.517(0.283-8.147)$ & 0.627 & $6.693(1.391-32.210)$ & 0.049 \\
Baseline laboratory values & & & & \\
IgG (mg/dL, $\geq 1,860 /<1,860)$ & $5.224 \times 10^{4}(0.000-$ Inf) & 0.021 & & 0.046 \\
\hline Platelets $\left(10^{4} / \mu \mathrm{L}, \geq 18 /<18\right)$ & $1.057(0.192-5.813)$ & 0.949 & $3.873(1.021-14.690)$ &
\end{tabular}

\section{Management and prognosis of DM in AlH}

DM was treated with biguanides in two cases, dipeptidyl peptidase-4 inhibitors in 10 cases, sulfonylureas in four cases and insulin in five cases; a pioglitazone and glinide were administered in one case each. Two or more classes of drugs were administered in four patients.

Seven patients died during the observation period. Death occurred at a median of 9.5 years after diagnosis (range, 518 years) at a median age of 75.0 years (range, 66-83 


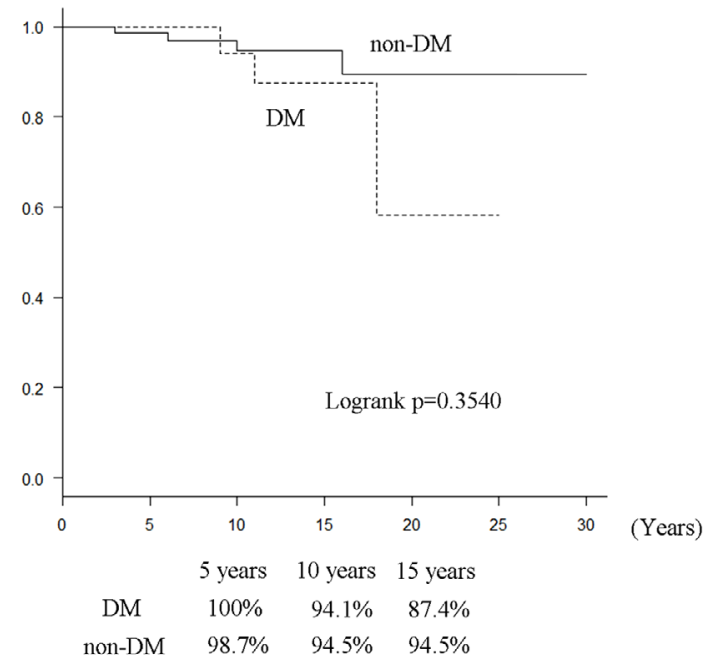

Figure 4. Cumulative survival curves for the DM and nonDM groups. The 10-year survival rates of the DM and non-DM groups were $94.1 \%$ and $94.6 \%$, respectively. No significant difference was observed between the two groups.

years). The 10 year survival rates of the DM and non-DM groups were $94.1 \%$ and $94.6 \%$, respectively. There was no significant difference between the DM and non-DM groups $(\mathrm{p}=0.293)$ (Fig. 4). The causes of death were hepatocellular carcinoma in four cases and liver failure, sepsis and pneumonia in one case each.

\section{Discussion}

In the present study, the prevalence of DM in patients with AIH was $24.5 \%$. The risk factors of DM were advanced age and a higher serum immunoglobulin $G$ level. The predictive factors for a new onset of DM were consumption of corticosteroids, absence of other autoimmune diseases and a lower platelet count. The prognosis of AIH was not affected by DM. This study is the first to report the independent factors associated with DM in AIH.

The liver is the main organ associated with carbohydrate metabolism through gluconeogenesis and glycogenolysis. Hepatic dysfunction leads to glucose abnormalities, especially in chronic liver disease $(8,9)$. Various chronic liver diseases are associated with DM $(8,9)$. Many studies report that HCV increases the incidence of DM (13-17). The hepatitis $C$ virus induces DM through insulin resistance via a variety of direct and indirect mechanisms (12). An estimated 1.4-46.7\% of patients infected with $\mathrm{HCV}$ also have DM, and a meta-analysis shows $\mathrm{HCV}$ infection increases the risk of developing DM by approximately 1.7 -fold compared to that in non-infected controls (16). Diabetes mellitus also occasionally occurs in $\mathrm{HBV}$-infected patients; previous studies indicate the prevalence rate of DM is $6.3-12 \%$ in $\mathrm{HBV}$ infection $(13-15,18)$. However, a recent population-based cohort study suggested that HBV infection had no effect on DM development (27). Moreover, the prevalence of DM in alcoholic liver disease and NAFLD ranges from 7.4 to
$19 \%(11,19)$ and 2.5 to $22.5 \%(11,20)$, respectively.

However, there are few reports in the literature about the prevalence and risk factors of DM in patients with AIH. A previous study from Sweden indicates that $8.7 \%$ of patients with AIH had DM (3), which is a lower incidence rate than that of the present study. The difference between these studies can be explained by the fact that the patients in the previous study were younger than our patients. Another study reports the incidence rate of DM in Japanese patients with AIH to be $15.0 \%$ (28), which is similar to the present study. The risk factors of DM onset in patients with chronic liver disease are reported to be male sex, advanced age and the presence of cirrhosis $(13,14,27,29)$. The present study revealed that advanced age, male sex and corticosteroid use were risk factors of DM (Table 3). These results suggest that liver fibrosis does not influence the onset of DM in AIH. We considered that aging might influence the elevation of HbAlc at nine years after baseline (Fig. 3). Advanced age as a risk factor is consistent with the conclusions of published studies, and corticosteroid use, absence of other autoimmune diseases and higher serum immunoglobulin G levels are characteristic causes of AIH. Yoneyama et al. administered corticosteroids to 29 patients with $\mathrm{AIH}$, and 11 experienced the onset of DM (6).

Our data revealed that the presence of other autoimmune diseases lowered the incidence of DM. The development of type $2 \mathrm{DM}$ has been considered to not have an autoimmune component (30). A recent study showed gene variants predisposing patients to autoimmune diseases are not associated with the risk of developing type 2 diabetes (31). Although various antibodies such as anti-liver kidney microsomal antibodies (anti-LKM antibody) or anti-centromere antibody may be associated with DM, they were examined in only a small number of cases in this study. The association between the absence of autoimmune disease and onset of DM is unknown. The influence of immunoglobulin G on DM is also unclear. A recent study revealed that a lower serum immunoglobulin $\mathrm{G}$ level was associated with the onset of nondiabetic renal disease in patients with type 2 DM (32). It is necessary to consider autoimmune hepatitis induced by antidiabetic drugs (33). However, it is important to note that there are no published studies describing autoimmune phenomena caused by antidiabetic agents.

The standard treatments for DM with chronic liver diseases are metformin and insulin (34). Metformin is a firstline therapy for type $2 \mathrm{DM}$ in obese patients. Although metformin occasionally induces lactic acidosis, this appears to be most prevalent in patients with cirrhosis and current alcohol overuse (35). Metformin is otherwise likely to be reasonably safe. Despite an increased risk of hypoglycemia, insulin therapy is safe and the most effective antihyperglycemic therapy for patients with chronic liver disease (36). Dipeptidyl peptidase-4 inhibitors are a new class of hypoglycemic drugs that reduce glucagon and blood glucose levels by increasing incretin levels. However, their efficacy and safety remain unknown. The prognosis of AIH with appro- 
priate treatment is good, and our data suggest DM worsens in such patients. We believe it is more important to administer antihyperglycemic drugs than reduce or withdraw corticosteroids, because insufficient corticosteroid use can lead to hepatic inflammation relapse.

There are several limitations associated with this study. First, more than half of the AIH subjects did not undergo a liver biopsy. Autoimmune hepatitis was diagnosed according to the 1999 revised criteria. Second, this was a single-center study and therefore requires external validation. Third, oral glucose tolerance tests were not performed in most patients. Although the oral glucose tolerance test is adequate for precisely diagnosing type $2 \mathrm{DM}$, it is still not routine in $\mathrm{AIH}$ subjects because of increased cost and inconvenience. The main strength of the present study is the long follow-up period.

In conclusion, DM occurred in $24.5 \%$ of patients with $\mathrm{AIH}$, and male sex, absence of other autoimmune diseases and a higher serum immunoglobulin $\mathrm{G}$ level are risk factors for DM. Taking corticosteroids and a lower platelet count are risk factors for a new onset of DM.

The authors state that they have no Conflict of Interest (COI).

\section{References}

1. Waldenström J. Leber, Blutproteine und Nahrungseiweiss. Dtsh Ges Verdau Stoffwechselkr 15: 113-119, 1950.

2. Soloway RD, Summerskill WH, Baggenstoss AH, et al. Clinical, biochemical, and histological remission of severe chronic active liver disease: a controlled study of treatments and early prognosis. Gastroenterology 63: 820-833, 1972.

3. Werner $M$, Wallerstedt $S$, Lindgren $S$, et al. Characteristics and long-term outcome of patients with autoimmune hepatitis related to the initial treatment response. Scand J Gastroenterol 45: 457467, 2010.

4. Murray-Lyon IM, Stern RB, Williams R. Controlled trial of prednisone and azathioprine in active chronic hepatitis. Lancet 1: 735737, 1973.

5. Cook GC, Mulligan R, Sherlock S. Controlled prospective trial of corticosteroid therapy in active chronic hepatitis. Q J Med 40: 159-185, 1971.

6. Yoneyama K, Honda E, Kogo M, et al. Efficacy and safety of prednisolone in patients with autoimmune hepatitis. Adv Ther 23: 74-91, 2006.

7. Frauman AG. An overview of the adverse reactions to adrenal corticosteroids. Adverse Drug React Toxicol Rev 15: 203-206, 1996.

8. Long RG. Endocrine aspects of liver disease. Br Med J 280: 225$228,1980$.

9. Kingston ME, Ali MA, Atiyeh M, et al. Diabetes mellitus in chronic active hepatitis and cirrhosis. Gastroenterology 87: 688694, 1984.

10. La Vecchia C, Negri E, Decarli A, et al. Diabetes mellitus and the risk of primary liver cancer. Int J Cancer 73: 204-207, 1997.

11. Stepanova M, Rafiq N, Younossi ZM. Components of metabolic syndrome are independent predictors of mortality in patients with chronic liver disease: a population-based study. Gut 59: 1410$1415,2010$.

12. Bugianesi E, Salamone F, Negro F. The interaction of metabolic factors with HCV infection: does it matter? J Hepatol 56 (Suppl 1): S56-S65, 2012.
13. Mason AL, Lau JY, Hoang N, et al. Association of diabetes mellitus and chronic hepatitis C virus infection. Hepatology 29: 328333, 1999.

14. Arao M, Murase K, Kusakabe A, et al. Prevalence of diabetes mellitus in Japanese patients infected chronically with hepatitis C virus. J Gastroenterol 38: 355-360, 2003.

15. Imazeki F, Yokosuka O, Fukai K, et al. Prevalence of diabetes mellitus and insulin resistance in patients with chronic hepatitis $\mathrm{C}$ : comparison with hepatitis B virus-infected and hepatitis C viruscleared patients. Liver Int 28: 355-362, 2008.

16. White DL, Ratziu V, El-Serag HB. Hepatitis $C$ infection and risk of diabetes: a systematic review and meta-analysis. J Hepatol 49: 831-844, 2008.

17. Arase Y, Suzuki F, Suzuki Y, et al. Sustained virological response reduces incidence of onset of type 2 diabetes in chronic hepatitis C. Hepatology 49: 739-744, 2009.

18. Huang ZS, Huang TS, Wu TH, et al. Asymptomatic chronic hepatitis B virus infection does not increase the risk of diabetes mellitus: a ten-year observation. J Gastroenterol Hepatol 25: 14201425, 2010.

19. Zein NN, Abdulkarim AS, Wiesner RH, et al. Prevalence of diabetes mellitus in patients with end-stage liver cirrhosis due to hepatitis C, alcohol, or cholestatic disease. J Hepatol 32: 209-217, 2000.

20. Targher G, Byrne CD. Clinical review: Nonalcoholic fatty liver disease: a novel cardiometabolic risk factor for type 2 diabetes and its complications. J Clin Endocrinol Metab 98: 483-495, 2013.

21. Hickman IJ, Macdonald GA. Impact of diabetes on the severity of liver disease. Am J Med 120: 829-834, 2007.

22. Matsumoto N, Arase Y, Seko Y, et al. Prevalence and predictive factors of diabetes in hepatitis virus positive liver cirrhosis with fasting plasma glucose level of $<126 \mathrm{mg} / \mathrm{dL}$. Hepatol Res 42: 558563, 2012.

23. Alvarez F, Berg PA, Bianchi FB, et al. International Autoimmune Hepatitis Group Report: review of criteria for diagnosis of autoimmune hepatitis. J Hepatol 31: 929-938, 1999.

24. Chazouillères O, Wendum D, Serfaty L, et al. Primary biliary cirrhosis-autoimmune hepatitis overlap syndrome: clinical features and response to therapy. Hepatology 28: 296-301, 1998.

25. Calès P, Oberti F, Michalak S, et al. A novel panel of blood markers to assess the degree of liver fibrosis. Hepatology 42: 13731381, 2005.

26. The French METAVIR Cooperative Study Group. Intraobserver and interobserver variations in liver biopsy interpretation in patients with chronic hepatitis C. Hepatology 20: 15-20, 1994.

27. Spradling PR, Simons B, Narayanan M, et al. Incidence of diabetes mellitus in a population-based cohort of persons with chronic hepatitis B virus infection. J Viral Hepat 20: 510-513, 2013.

28. Hino-Arinaga T, Ide T, Kuromatsu R, et al; Autoimmune Hepatitis Study Group. Risk factors for hepatocellular carcinoma in Japanese patients with autoimmune hepatitis type 1 . J Gastroenterol 47: 569-576, 2012.

29. Petit JM, Bour JB, Galland-Jos C, et al. Risk factors for diabetes mellitus and early insulin resistance in chronic hepatitis C. J Hepatol 35: 279-283, 2001.

30. Brooks-Worrell B, Palmer JP. Immunology in the Clinic Review Series; focus on metabolic diseases: development of islet autoimmune disease in type 2 diabetes patients: potential sequelae of chronic inflammation. Clin Exp Immunol 167: 40-46, 2012.

31. Rafiq S, Melzer D, Weedon MN, et al. Gene variants influencing measures of inflammation or predisposing to autoimmune and inflammatory diseases are not associated with the risk of type 2 diabetes. Diabetologia 51: 2205-2213, 2008.

32. Weng $\mathrm{CH}, \mathrm{Hu} \mathrm{CC}, \mathrm{Yu} \mathrm{CC}$, et al. Immunoglobulin $\mathrm{G}$ levels can predict non-diabetic renal disease in patients with type 2 diabetes 
mellitus. J Diabetes 4: 37-40, 2012.

33. Björnsson E, Talwalkar J, Treeprasertsuk S, et al. Drug-induced autoimmune hepatitis: clinical characteristics and prognosis. Hepatology 51: 2040-2048, 2010.

34. Custro N, Carroccio A, Ganci A, et al. Glycemic homeostasis in chronic viral hepatitis and liver cirrhosis. Diabetes Metab 27 (4 Pt
1): 476-481, 2001.

35. Khan R, Foster GR, Chowdhury TA. Managing diabetes in patients with chronic liver disease. Postgrad Med 124: 130-137, 2012.

36. Brackett CC. Clarifying metformin's role and risks in liver dysfunction. J Am Pharm Assoc (2003) 50: 407-410, 2010.

\footnotetext{
(C) 2016 The Japanese Society of Internal Medicine http://www.naika.or.jp/imonline/index.html
} 